\title{
Research on Constant Force Polishing Control of Aero-engine Blade Based on Expanded State Observer
}

\section{Wenhua Zhang}

Hebei Industrial University: Hebei University of Technology

Shijie Dai ( $\nabla$ dshj70@163.com )

Hebei University of Technology https://orcid.org/0000-0002-9082-9984

\section{Yufeng Zhao}

Hebei University of Technology

Jiaheng Mu

Hebei University of Technology

Tianrong Hu

Hebei University of Technology

\section{Pengwei Li}

Hebei University of Technology

\section{Riqing Deng}

Hebei University of Technology

\section{Lei Yan}

Hebei University of Technology

\section{Research Article}

Keywords: Aero-engine blade, Robot, ADRC, SMC, ESO, Constant force control

Posted Date: August 17th, 2021

DOI: https://doi.org/10.21203/rs.3.rs-774635/v1

License: (1) (1) This work is licensed under a Creative Commons Attribution 4.0 International License. Read Full License 


\title{
Research on constant force polishing control of
}

\section{aero-engine blade based on expanded state observer}

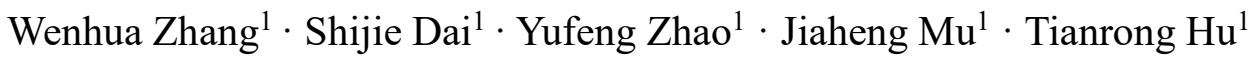 \\ Pengwei $\mathrm{Li}^{1}$ - Riqing Deng ${ }^{1} \cdot$ Lei Yan ${ }^{1}$ \\ ${ }^{1}$ School of Mechanical Engineering, Hebei University of Technology, Tianjin, 300401, \\ PR China
}

Corresponding author: Shijie Dai (E-mail address: dshj70@163.com)

\begin{abstract}
This paper presents a parallel control method based on the expanded state observer (ESO) for aero-engine blade robot polishing. Aiming to reduce the fluctuation of polishing force caused by environmental noise and modeling errors. First, calibrate six-dimensional force sensor according to the maximum acceleration of the end effector during the polishing process. Then, build the gravity compensation and zero drift compensation model. Besides, use this model to compensate measurement error of the six-dimensional force sensor. Finally, calculate the error between the expected polishing force and the actual feedback value and its derivative value. Use calculation results to design the control boundary layer. The polishing force controller is divided into two parallel control loops to design. When the switching value is in the control boundary layer. A nonlinear active disturbance rejection control (ADRC) loop is used. When the switching value is outside the control boundary layer. An ESO-based sliding mode control (SMC) loop is used. Simulation and experimental results show that the proposed parallel control method based on ESO has a fast response and high robustness compared with FuzzyPID, PID, and ADRC. It can effectively suppress the force fluctuation in the polishing process and significantly improve the surface processing quality of the aero-engine blade.
\end{abstract}

Keywords: Aero-engine blade $\cdot$ Robot $\cdot$ ADRC $\cdot$ SMC $\cdot$ ESO $\cdot$ Constant force control

\section{Introduction}

Aero-engine blades have a long time been exposed to harsh working conditions of high temperature, high pressure, high speed, and alternating loads. It is easy to produce defects such as fatigue cracks and ablation. So has extremely high requirements for manufacturing accuracy and processing [1-3]. At present, a variety of special processing methods have been applied in aero-engine blade grinding and polishing. For example, electrolytic polishing has been used on hard and brittle materials. It can effectively improve the material removal rate and reduce the polishing wheel rate of wear [4-5]. But those techniques are mainly used for rough machining [6]. Manual polishing is difficult to ensure the surface quality and polishing efficiency of the 
aero-engine blade. Similarly, computer-numerical control (CNC) not only has poor flexibility but also is expensive [7]. Therefore, many scholars have devoted themselves to the work of robotic polishing and polishing technology [8]. For example, Zhang et al. [9] developed an abrasive belt mechanism. That can automatically adjust the polishing force between the end polishing tool and the aero-engine blade surface. Chen et al. [10] designed a contour-adaptive and active compliant polishing end effector. Chen et al. [11] proposed a two degree of freedom active compliant end device that can automatically adjust to change in curvature. However, control the polishing force of the robot is always a difficult problem in the field of robot force control [12]. The imperfect force control measures of the polishing robot can easily lead to the problem of overpolishing or under-polishing [13-14]. Over-polishing not only damages the end effector but also reduces the quality of an aero-engine blade or even destroys it [15]. Underpolishing requires constant feedback adjustment resulting in low polishing efficiency. Therefore, it has significance to study the constant polishing of aero-engine blades.

In view of the problem of polishing force fluctuation control in the polishing process. Some scholars consider the contact stiffness between the robot and the environment. For example, Loris et al. [16] summarized the defects of the existing two force tracking methods based on impedance control. Set-point deformation and variable stiffness approaches. Proposed to adopt extended Kalman filter online estimation of contact stiffness between the robot and the environment. However, the desired effect is still not achieved. So many scholars are dedicated to the study of error feedback control based on the expected value and the actual value of the polishing force. Loris et al. [17] proposed a method combined iterative learning with reinforcement learning algorithms to achieve adaptive adjustment control. Xu et al. [18] proposed a method that combined force-position hybrid control with PI/PD control. Du et al. [19] designed an adaptive anti-saturation integral separation fuzzy proportional-integral controller. Xiao et al. [20] developed constant-load adaptive belt polishing technology based on force-position hybrid control for thin-walled weak rigidity blisk blade. Although the current research based on error feedback control research has been deepened. But only a few researchers have considered real-time compensation for environmental noise and modeling errors. And apply force control method after implementing polishing force error feedback control during the polishing process. Such as Niu et al. [21] proposed a feed-forward compensation method based on disturbance observer and PI feedback control to keep the current constant in the raymond mill polishing process. Philippe et al. [22-23] proposed to improve the robustness of the system controller based on the state observer and the disturbance observer. Then proposed a multi-objective genetic algorithm to be apply to the underwater polishing and polishing robot based on his previous research. Aiming at the control of the ball mill polishing circuits. Chen et al. [24] proposed a method of fusion observer feed-forward compensation combined with PI feedback control. Later, Yang et al. [25] proposed a method that combined feed-forward compensation based on disturbance observer with feedback control based on model prediction. Although the above-mentioned scholars considered the influence of environmental noise and modeling error in the research process. And subsequently proposed to optimize the controller parameters based on the optimization algorithm to 
improve the control robustness. But in the actual processing process, the environmental disturbance is time-varying. Environmental disturbance and modeling error causes the fluctuation of polishing force also is time-varying.

So further improvement and optimization are needed. Improve the robustness and response speed of system control. Therefore, a parallel control method based on the expanded state observer (ESO) is proposed to improve the robustness and rapid response of the controller. Realize the constant-force polishing control of the aero-engine blade. Improve the surface polishing quality of the aero-engine blade.

\section{Polishing force/torque perception}

Accurate measurement of the polishing force between the blades and the end effector is the basis to ensure constant polishing. The correct six-dimensional force sensor must be selected to prevent damage to the blades due to polishing force perception errors. In addition, the gravity of the end effector and the zero drift of the six-dimensional force sensor will cause greater errors in the perception of polishing force. Therefore, it must be the gravity compensation and zero drift compensation before processing.

\subsection{Calibration of six-dimensional force sensor}

In order to choose the correct calibration method, it is necessary to consider the maximum force and torque applied to the sensor. In the working environment of the robot applications, the maximum load of the six-dimensional force sensor is the dynamic load. It caused by the acceleration of the end effector of the robot. So use acceleration sensor to measure the maximum acceleration of the end effector. And correct calibration method of the six-dimensional force sensor to be determined.

The maximum dynamic torque that the six-dimensional force sensor needs to endure can be expressed as:

$$
T_{\max }=m \times\left(g+a_{\max }\right) \times x
$$

Where $m$ represents the mass of the end effector, $g$ represents the acceleration of gravity, $a_{\text {max }}$ represents the maximum acceleration of the end effector. $x$ represents the distance from the center of mass of the end effector to the upper surface of the six-dimensional force sensor. The calculation of center of mass of the end effector is described in section 3. As shown in Fig. 1, the relationship of robotic coordinate systems. Where $\left\{O X_{0} Y_{0} Z_{0}\right\}$ is the world coordinate frame. $\left\{O X_{1} Y_{1} Z_{1}\right\}$ is the robot flange coordinate frame. $\left\{O X_{2} Y_{2} Z_{2}\right\}$ is the six-dimensional force sensor coordinate frame. $\left\{O X_{3} Y_{3} Z_{3}\right\}$ is the end effector coordinate frame. 


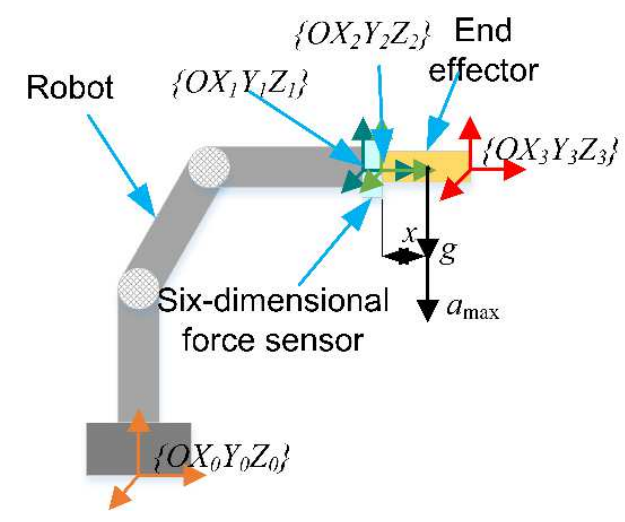

Fig. 1 The relationship of robotic coordinate systems

\subsection{Six-dimensional force sensor gravity compensation and zero drift}

\section{compensation}

When the six-dimensional force sensor is under no load only the gravity of the end effector is considered. The polishing force/torque measured by the six-dimensional force sensor is expressed in the coordinate system $\left\{\mathrm{OX}_{2} Y_{2} Z_{2}\right\}$ as:

$$
{ }^{2} \mathbf{G}=\left\{\begin{array}{llllll}
{ }^{2} F_{g x} & { }^{2} F_{g y} & { }^{2} F_{g z} & { }^{2} T_{g x} & { }^{2} T_{g y} & { }^{2} T_{g z}
\end{array}\right\}
$$

In the initial state of aero-engine blade polishing, it is assumed that the tilt sensor coincides with the world coordinate system $\left\{O X_{0} Y_{0} Z_{0}\right\}$. In the polishing process, the spatial attitude of the end effector needs to be constantly changed to adapt to the change of the blade curvature. During the experiment, a three-axis dynamic tilt angle sensor is used to measure the pitch angle $\beta_{p}$, roll angle $\beta_{r}$, and yaw angle $\beta y$. These angles represent the six-dimensional force sensor coordinate system relative to the world coordinate system in real time. Assuming that the coordinate system $\left\{O X_{1} Y_{1} Z_{1}\right\}$ and the coordinate system $\left\{\mathrm{OX}_{2} Y_{2} Z_{2}\right\}$ have the same coordinate axis direction. Then calculate the gravity component of the end effector in the coordinate frame $\left\{\mathrm{OX}_{2} Y_{2} Z_{2}\right\}$. Through the conversion relationship between the world coordinate frame $\left\{O X_{0} Y_{0} Z_{0}\right\}$ and the coordinate frame $\left\{\mathrm{OX}_{2} Y_{2} Z_{2}\right\}$.

The conversion relationship between the coordinate frame $\left\{O X_{0} Y_{0} Z_{0}\right\}$ and the coordinate frame $\left\{\mathrm{OX}_{2} \mathrm{Y}_{2} \mathrm{Z}_{2}\right\}$ can be expressed as:

$$
\begin{aligned}
& { }_{0}^{2} \boldsymbol{R}=\boldsymbol{R}\left(x, \beta_{p}\right) \boldsymbol{R}\left(y, \beta_{r}\right) \boldsymbol{R}\left(z, \beta_{y}\right) \\
& =\left[\begin{array}{ccc}
1 & 0 & 0 \\
0 & \cos \beta_{p} & -\sin \beta_{p} \\
0 & \sin \beta_{p} & \cos \beta_{p}
\end{array}\right]\left[\begin{array}{ccc}
\cos \beta_{r} & 0 & \sin \beta_{r} \\
0 & 1 & 0 \\
-\sin \beta_{p} & 0 & \cos \beta_{p}
\end{array}\right]\left[\begin{array}{ccc}
\cos \beta_{y} & 0 & -\sin \beta_{y} \\
\sin \beta_{y} & 0 & \cos \beta_{y} \\
0 & 0 & 1
\end{array}\right] \\
& =\left[\begin{array}{ccc}
c \beta_{r} \cdot c \beta_{y} & -c \beta_{r} \cdot s \beta_{y} & s \beta_{r} \\
s \beta_{p} \cdot s \beta_{r} \cdot c \beta_{y}+c \beta_{p} \cdot s \beta_{y} & -s \beta_{p} \cdot s \beta_{r} \cdot s \beta_{y}+c \beta_{p} \cdot c \beta_{y} & -s \beta_{p} \cdot c \beta_{r} \\
-c \beta_{p} \cdot s \beta_{r} \cdot c \beta_{y}+s \beta_{p} \cdot s \beta_{y} & c \beta_{p} \cdot s \beta_{r} \cdot s \beta_{y}+s \beta_{p} \cdot c \beta_{y} & c \beta_{p} \cdot c \beta_{y}
\end{array}\right]
\end{aligned}
$$

Where $s \beta_{i(i=p, r, y)}=\sin \beta_{i(i=p, r, y)}, \quad c \beta_{i(i=p, r, y)}=\cos \beta_{i(i=p, r, y)}$. 
In the static state and without additional load, the gravity $G$ of the end effector is expressed in the world coordinate frame as:

$$
{ }^{\mathbf{0}} \mathbf{G}=\left[\begin{array}{llllll}
0 & 0 & -G & 0 & 0 & 0
\end{array}\right]
$$

Assumptions:

(1) The coordinate of the center of mass of the end effector in coordinate frame $\left\{O X_{2} Y_{2} Z_{2}\right\}$ is $\left({ }^{2} x,{ }^{2} y,{ }^{2} z\right)$.

(2) When there is no additional load, consider the end effector gravity and zero drift errors. The actual measured value of the force/torque of the six-dimensional force sensor in coordinate frame $\left\{\mathrm{OX}_{2} Y_{2} Z_{2}\right\}$ is expressed as:

$$
\left\{\begin{array}{llllll}
{ }^{2} F_{x} & { }^{2} F_{y} & { }^{2} F_{z} & { }^{2} T_{x} & { }^{2} T_{y} & { }^{2} T_{z}
\end{array}\right\} .
$$

According to the above assumptions.

$$
\left[\begin{array}{c}
{ }^{2} F_{g x} \\
{ }^{2} F_{g y} \\
{ }^{2} F_{g z} \\
{ }^{2} T_{g x} \\
{ }^{2} T_{g y} \\
{ }^{2} T_{g z}
\end{array}\right]=\left[\begin{array}{ccc}
1 & 0 & 0 \\
0 & 1 & 0 \\
0 & 0 & 1 \\
0 & { }^{2} z & 2 y \\
{ }^{2} z & 0 & { }^{2} x \\
{ }^{2} y & { }^{2} x & 0
\end{array}\right]\left[\begin{array}{l}
{ }^{2} F_{g x} \\
{ }^{2} F_{g y} \\
{ }^{2} F_{g z}
\end{array}\right]
$$

The actual measurement value of the six-dimensional force sensor includes the zero drift error.

$$
\left[\begin{array}{c}
{ }^{2} F_{x} \\
{ }^{2} F_{y} \\
{ }^{2} F_{z} \\
{ }^{2} T_{x} \\
{ }^{2} T_{y} \\
{ }^{2} T_{z}
\end{array}\right]=\left[\begin{array}{ccc}
1 & 0 & 0 \\
0 & 1 & 0 \\
0 & 0 & 1 \\
0 & { }^{2} z & 2 \\
{ }^{2} z & 0 & { }^{2} x \\
{ }^{2} y & { }^{2} x & 0
\end{array}\right]\left[\begin{array}{c}
{ }^{2} F_{g x} \\
{ }^{2} F_{g y} \\
{ }^{2} F_{g z}
\end{array}\right]+\left[\begin{array}{c}
{ }^{2} F_{x 0} \\
{ }^{2} F_{y 0} \\
{ }^{2} F_{z 0} \\
{ }^{2} T_{x 0} \\
{ }^{2} T_{y 0} \\
{ }^{2} T_{z 0}
\end{array}\right]
$$

Where ${ }^{2} F_{x 0},{ }^{2} F_{y 0},{ }^{2} F_{z 0},{ }^{2} T_{x 0},{ }^{2} T_{y 0},{ }^{2} T_{z 0}$ indicates the zero point drift value of each axis force/torque of the six-dimensional force sensor.

Simplification can get the following formula:

$$
\left[\begin{array}{c}
{ }^{2} T_{x} \\
{ }^{2} T_{y} \\
{ }^{2} T_{z}
\end{array}\right]=\left[\begin{array}{lll}
{ }^{2} F_{x}-{ }^{2} F_{x 0} & { }^{2} F_{y}-{ }^{2} F_{y 0} & { }^{2} F_{z}-{ }^{2} F_{z 0}
\end{array}\right]\left[\begin{array}{c}
{ }^{2} x \\
2 \\
y \\
{ }^{2} z
\end{array}\right]+\left[\begin{array}{c}
{ }^{2} T_{x 0} \\
{ }^{2} T_{y 0} \\
{ }^{2} T_{z 0}
\end{array}\right]
$$

When the robot is in static state, control the robot to realize the measurement of force and torque signals in different postures $N$ times. To avoid measurement errors, make sure $N \geq 3$. Besides, make the direction of the end effector of the robot should not be in the same horizontal plane each time. Using the collected forces and torques, the position 
coordinates of the center of mass of the end effector in the coordinate frame $\left\{\mathrm{OX}_{2} \mathrm{Y}_{2} \mathrm{Z}_{2}\right\}$ can be calculated. However, due to the continuous adjustment of the end effector posture during the actual polishing process, the center of mass is constantly changing in coordinate frame $\left\{O X_{0} Y_{0} Z_{0}\right\}$. Assuming that the displacement changes of the end effector along the coordinate axis $O X, O Y, O Z$ in the coordinate frame $\left\{O X_{0} Y_{0} Z_{0}\right\}$ are $\left(\begin{array}{lll}\Delta x & \Delta y & \Delta z\end{array}\right)$ respectively. The real-time coordinates of the center of mass of the end effector in the coordinate frame $\left\{O X_{0} Y_{0} Z_{0}\right\}$ can be obtained as:

$$
\left[\begin{array}{c}
x \\
y \\
z
\end{array}\right]={ }_{0}^{2} \boldsymbol{R}^{-1}\left[\begin{array}{c}
2 \\
2 \\
y \\
{ }^{2} z
\end{array}\right]+\left[\begin{array}{c}
\Delta x \\
\Delta y \\
\Delta z
\end{array}\right]
$$

During the polishing process, the actual polishing force between the end effector and the aero-engine blade can be calculated as:

$$
{ }^{2} F_{c 1}={ }^{2} F_{m}-{ }^{2} F_{g}-{ }^{2} F_{0}={ }^{2} F_{m}-\left[\begin{array}{c}
{ }^{2} F_{x} \\
{ }^{2} F_{y} \\
{ }^{2} F_{z}
\end{array}\right]
$$

Where ${ }^{2} F_{m}$ represents the force signal actually measured by the six-dimensional force sensor. ${ }^{2} F_{c 1}$ indicates the polishing force during the actual polishing process.

The compensation results of the six-dimensional force sensor using gravity compensation and zero-point drift methods are shown in Fig. 2. When without additional load, the average value of the six-dimensional force sensor perception error is $0.2812 \mathrm{~N}$ and the variance is 0.4341 after compensation, the polishing force can be better measured.

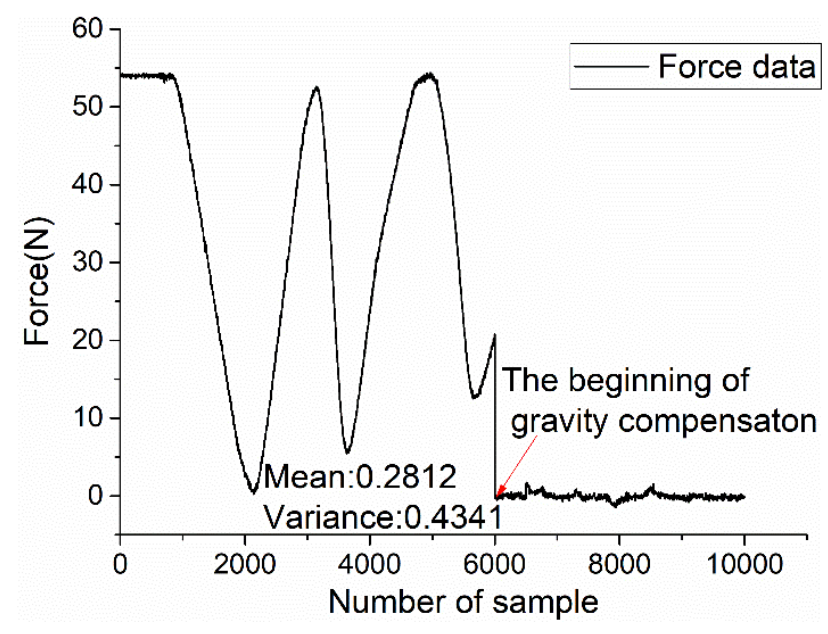

Fig. 2 Polishing force after compensation

\section{Control system design}

During the polishing process of the aero-engine blade, due to environmental disturbance and modeling errors, could resulting in polishing force fluctuate. The active disturbance rejection control (ADRC) is used to realize the polishing force control. But due to the limitation of the extended state observer (ESO) observation range. Result in the robustness of the controller is insufficient. Therefore, the sliding mode control 
(SMC) is used when the environmental noise frequency is high. The SMC will vibrate near the switching surface, resulting in a sharply decrease of polishing quality and end effector service life. In order to improve the robustness of the controller while avoiding high-frequency vibrations. Propose a parallel control method of SMC and ADRC based on ESO (SMC+ADRC). A control boundary error layer is introduced. When the error is outside the control boundary error layer, ESO-based SMC is used. And the error is within the control boundary error layer ADRC is used.

\subsection{Design of ADRC}

In order to ensure the actual polishing force from zero initial value to the desired value. Realize rapid transition and avoid overshoot, at the same time to reduce the impact of environmental noise on the system. A tracking differentiator (TD) is used to achieve overshoot suppression and environmental noise filtering in the transition process. In addition, the method of predict first and then differentiate is adopted to solve the signal delay issue.

$$
\begin{aligned}
& \left\{\begin{array}{l}
d=r h_{0}^{2} \\
a_{0}=h_{0} F_{2} \\
y=F_{1}+a_{0} \\
\left.a_{1}=\sqrt{d(d+8|y|}\right) \\
a_{2}=a_{0}+\frac{\operatorname{sign}(y)\left(a_{1}-d\right)}{2} \\
a=\left(a_{0}+y\right) f_{s} g(y, d)+a_{2}\left(1-f_{s g}(y, d)\right) \\
\text { fhan }=-r\left(\frac{a}{d}\right) f \operatorname{ssg}(a, d)-r \operatorname{sign}(a)\left(1-f_{s g}(a, d)\right)
\end{array}\right. \\
& \left\{\begin{array}{l}
F_{0}(k)=F_{d}(k)+w_{1}(k) \\
F_{q}(k)=F_{0}(k)+q F_{2}(k) \\
f h(k)=\operatorname{fhan}\left(F_{1}(k)-F_{q}(k), F_{2}(k), r_{0}, h_{0}\right) \\
F_{1}(k+1)=F_{1}(k)+h F_{2}(k) \\
F_{2}(k+1)=F_{2}(k)+h f h(k)
\end{array}\right.
\end{aligned}
$$

Where $F_{d}$ represents the desired polishing force. $w_{1}$ represents the environmental noise during the polishing process. $F_{0}$ represents the system input polishing force, $F_{1}$ represents the tracking output of the signal. $F_{2}$ represents the signal $F_{1}$ speed of change. $r_{0}$ is the adjustment factor of the tracking speed. $h$ is the system solution step length. $h_{0}$ is the filter factor. $q$ is the phase compensation parameter.

The ESO is used to observe and compensate the environmental noise and modeling errors in the polishing aero-engine turbine blade. Use $z_{1}$ to observe the real-time status of the output of the system. Use $z_{2}$ to observe the speed of the change of the output polishing force of the system. Use $z_{3}$ to observe the disturbance $f(k)$ of the system, such as environmental noise, modeling error, etc. The structure of ESO is as follows: 


$$
\left\{\begin{array}{l}
e(k)=z_{1}(k)-F_{c 1}(k) \\
f e(k)=f a l(e(k), 0.5, \delta) \\
f e_{1}(k)=f a l(e(k), 0.25, \delta) \\
z_{1}(k+1)=z_{1}(k)+h\left(z_{2}(k)-\beta_{01} e(k)\right) \\
z_{2}(k+1)=z_{2}(k)+h\left(z_{3}(k)-\beta_{02} f e(k)+b_{0} F_{\text {out }}(k)\right) \\
z_{3}(k+1)=z_{3}(k)+h\left(-\beta_{03} f e_{1}(k)\right)
\end{array}\right.
$$

Where $b, \beta_{01}, \beta_{02}, \beta_{03}, \delta$ are adjustable parameter of the ESO.

Due to the influence of errors such as environmental noise and modeling error, feedback control based on errors is applied. Traditional PID has obvious effects for linear systems, but it is not ideal for systems affected by disturbances. Fuzzy PID can reduce the control error, but it has the defect of slow response. Although the intelligent control has a good simulation effect, it is difficult to realize in the actual application process. Design active disturbance rejection nonlinear state error feedback (NLSEF) control. The control structure is as follows:

$$
\left\{\begin{array}{l}
e_{1}(k)=F_{1}(k)-z_{1}(k) \\
e_{2}(k)=F_{2}(k)-z_{2}(k) \\
F_{\text {out }}(k)=\text { fhan }\left(e_{1}(k), c e_{2}(k), r_{0}, h_{1}\right)
\end{array}\right.
$$

Where $e_{1}$ and $e_{2}$ represents the difference respectively between the value $F_{1}, F_{2}$ and the output value $z_{1}, z_{2}$ of the extended observer. $r_{0}$ represents the speed factor. $c$ represents the damping factor. $h_{1}$ represents the system feedback control filter factor. $F_{\text {out }}$ represents NLSEF controller output.

ADRC controller system output can be expressed as follows:

$$
F_{\text {adrc }}(k)=F_{\text {out }}(k)
$$

\subsection{ESO-based SMC Design}

According to the SMC variable structure principle. In the state space, the sliding mode accessibility condition only guarantees that a moving point from any position can reach the switching surface within a finite time. There are no restrictions on the specific trajectory of the approaching motion. The approaching law method can improve the dynamic quality of the approaching motion. Characteristic of SMC variable structure control is the introduction of a switching law. Which enables the structure of the system to be changed in real time according to the current state of the system.

Assuming that the actual polishing process model is:

$$
\left\{\begin{array}{l}
\mathbf{F}_{c}(k+1)=\mathbf{A F}_{c}(k)+\mathbf{B} u(k)+\mathbf{D} f(k) \\
\mathbf{F}_{c}(k) \in \mathbf{R}^{n}, u(k) \in \mathbf{R}^{m}, f(k) \in \mathbf{R}^{t}
\end{array}\right.
$$

Among them, $\mathbf{F}_{c}(k)$ represents the actual state of the system in step $k, \mathbf{A}, \mathbf{B}, \mathbf{D}$ is the coefficient matrix.

Assuming that environmental disturbance and modeling error meet the matching conditions. 


$$
\mathbf{D}=\mathbf{B} \ddot{\bar{D}}
$$

Where $\ddot{\overline{\mathbf{D}}}$ them are uncertain. Then the system can be written as:

$$
\left\{\begin{array}{l}
\mathbf{F}_{c}(k+1)=\mathbf{A} \mathbf{F}_{c}(k)+\mathbf{B} u(k)+\mathbf{B} \overline{\mathbf{D}} f(k) \\
\mathbf{F}_{c}(k) \in \mathbf{R}^{2}, u(k) \in \mathbf{R}, f(k) \in \mathbf{R}
\end{array}\right.
$$

In order to improve the response speed of the controller, the following exponential approach law is selected.

$$
\frac{s(k+1)-s(k)}{T}=-\varepsilon \operatorname{sgn}(s(k))-k_{h} s(k) \quad \varepsilon>0, k_{h}>0
$$

Where $s(k)$ represents the state of the SMC process. $\operatorname{sgn}(s(k))$ represents a sign function with the argument. $\varepsilon, k_{h}$ is an adjustable parameter.

Using exponential approach law, the sliding surface design is as follows:

$$
s(k)=\mathbf{C}_{e} \mathbf{E}(k)=\mathbf{C}_{e}\left(\mathbf{F}(k)-\mathbf{F}_{c}(k)\right)
$$

Where $\mathbf{C}_{e}=[c, 1], \quad \mathbf{E}=\left[\begin{array}{c}e_{1}(k) \\ e_{2}(k)\end{array}\right], \quad \mathbf{F}=\left[\begin{array}{c}F_{1}(k) \\ F_{2}(k)\end{array}\right], \quad \mathbf{F}_{c}=\left[\begin{array}{c}F_{c 1}(k) \\ F_{c 2}(k)\end{array}\right]$.

In order to achieve a better transition, the saturation function $\operatorname{sat}(s)$ is used instead of the sign function $\operatorname{sgn}(s)$.

$$
\text { sat }=\left\{\begin{array}{cc}
1 & s>\Delta \\
k s & |s|<\Delta \quad k_{1}=\frac{1}{\Delta} \\
-1 & s<-\Delta
\end{array}\right.
$$

Where $\Delta$ is the control boundary layer. $k_{1}$ is the adjustable parameter of the controller.

$$
\begin{aligned}
& c\left\{\frac{\left[F_{1}(k+1)-z_{1}(k+1)\right]-\left[F_{1}(k)-z_{1}(k)\right]}{h}\right\}+\left\{\frac{\left[F_{2}(k+1)-z_{2}(k+1)\right]-\left[F_{2}(k)-z_{2}(k)\right]}{h}\right\} \\
& =-\operatorname{Esat}(s(k))-k_{h} s(k)
\end{aligned}
$$

Based on the above analysis, the control law of SMC+ADRC is as follows.

While $s>\Delta, s<\Delta$,

$$
F_{\text {final }}(k)=\left(\mathbf{C}_{e} \mathbf{B}\right)^{-1}\left(\mathbf{C}_{e} \mathbf{F}_{c}(k)-\mathbf{C}_{e} \mathbf{A} \mathbf{Z}(k)-\mathbf{C}_{e} \mathbf{B} \overline{\mathbf{D}} f(k)-s(k)-h\left(-\varepsilon \operatorname{sgn}(s(k))-k_{h} s(k)\right)\right)
$$

While $|s|<\Delta$,

$$
F_{\text {final }}(k)=F_{\text {adrc }}(k)
$$


The output $F_{\text {final }}(k)$ of the system controller is converted into a control signal $u_{c}(k)$ by the transfer function. Input into the system to realize the constant force polishing of the aero-engine blade. The system disturbance and modeling error can be compensated in real time according to equation (24).

$$
u(k)=u_{c}(k)-\frac{z_{3}(k)}{b}
$$

In order to improve the robustness of the controller while avoiding high frequency vibration caused by SMC. Introduce the control boundary layer. Using ESO-based $\mathrm{SMC}$ when the controller state is outside the control boundary layer. Using ADRC when controller state $s(k)$ is within the control boundary layer. The system controller structure is shown in Fig. 3. The conversion function and the state space function is calculated according to [26].

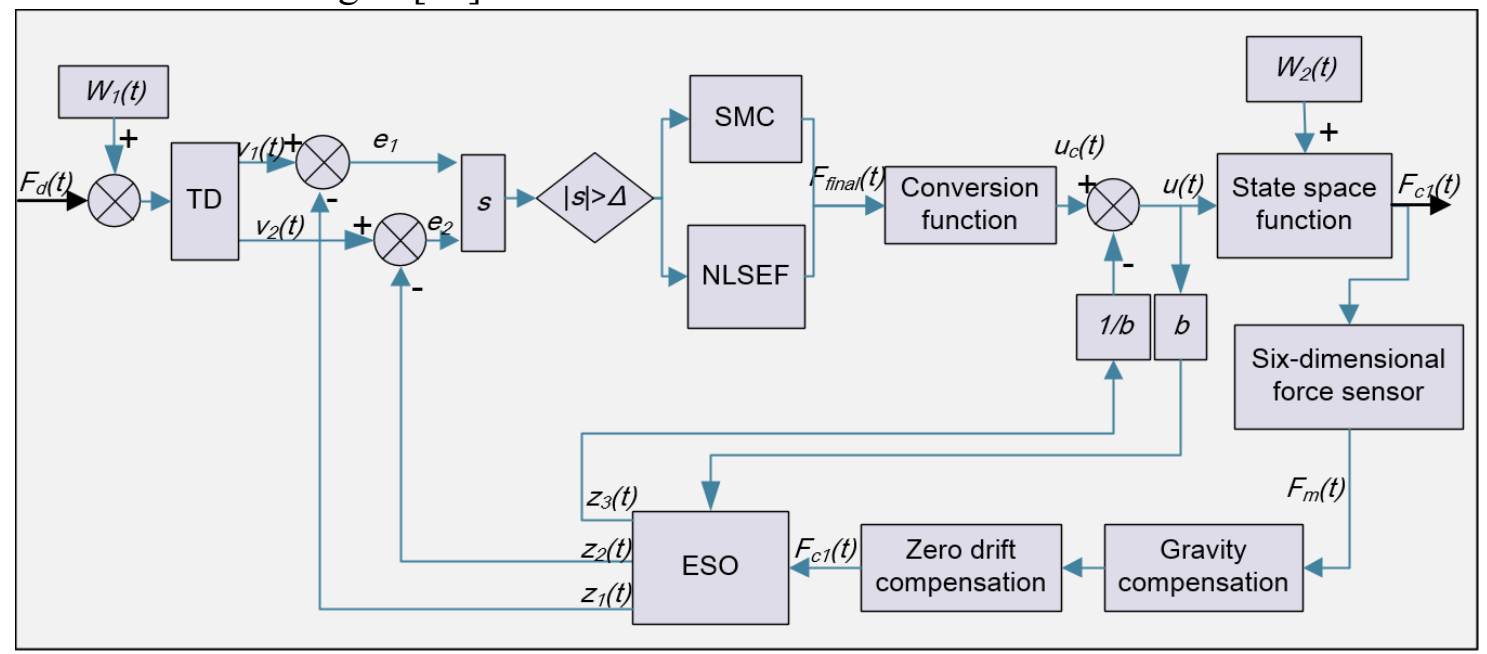

Fig. 3 structure of system controller

\section{Simulation and Experimental analysis}

End effector The TD realizes the initial filtering of the input signal $F_{0}$. The polishing force is quickly transitioned from the initial value of zero to the desired value. Meantime, polishing force overshoot is avoided. TD effectively solve the contradiction between rapid response and overshoot. In order to verify the effectiveness of TD filtering, add random Gaussian noise $w 1$ with a frequency of $1000 \mathrm{~Hz}$ to system. When the contact time is $0-2 \mathrm{~s}$, the expected polishing force is $8 \mathrm{~N}$. When contact time is $2-5 \mathrm{~s}$, the expected polishing force is $12 \mathrm{~N}$. In order to effectively solve the problem of TD signal phase lag. The method of prediction and then differentiation is applied. The simulation result of polishing force signal tracking is shown in Fig. 4. The force error range of TD output is $[-0.25,0.25]$. TD module parameters are shown in Table. 1 .

Table. 1 TD module parameters

\begin{tabular}{cccc}
\hline parameter & value & parameter & value \\
\hline$h_{0}$ & 0.06 & $r$ & 1500 \\
\hline$h$ & 0.001 & $q$ & 0.0015 \\
\hline
\end{tabular}




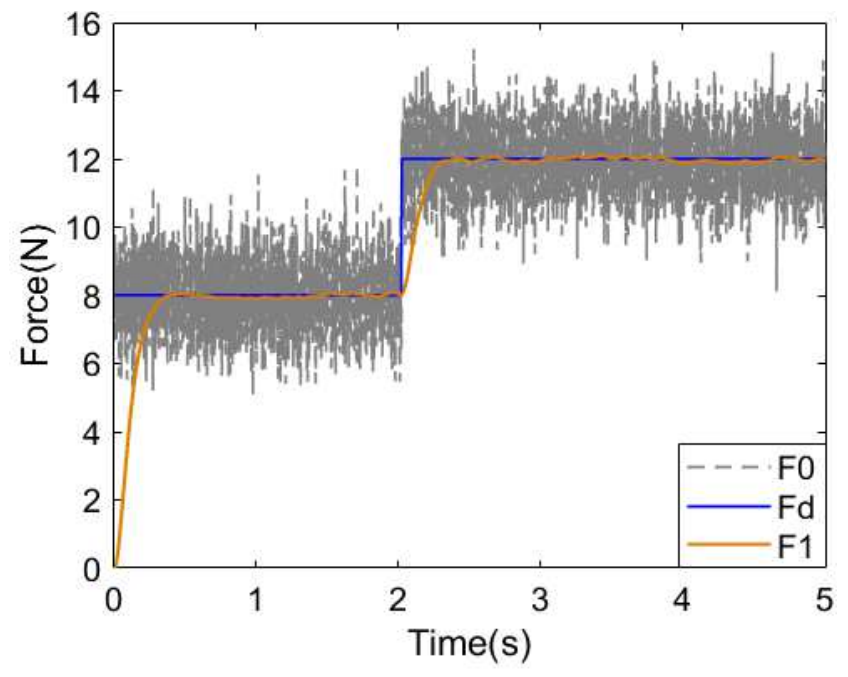

Fig. 4 Simulation of filtering effect of TD

In order to verify the effectiveness of ESO for observing disturbances in the machining process. A simulation study is carried out. Add the disturbance signal to system. Band-Limited White Noise $w_{2}$, the noise power is 0.01 , and the period is $0.05 \mathrm{~s}$. Fig. 5 shows a simulation result that using ESO to observe noise. This shows that the ESO can observe well the machining error in the aero-engine blade polishing process. Fig. 6 shows the comparison between the actual polishing force $F_{c 1}$ and the polishing force $z_{1}$ observed by ESO. Above results indicate that the ESO can achieve better tracking of the polishing force during the polishing process. The parameters of the ESO module are shown in Table. 2.

Table. 2 ESO module parameters

\begin{tabular}{cccc}
\hline parameter & value & parameter & value \\
\hline$h$ & 0.001 & $\beta_{01}$ & 1805 \\
$b_{0}$ & 0.5 & $\beta_{02}$ & 1080002 \\
$w_{o}$ & 600 & $\beta_{02}$ & 216000006 \\
\hline
\end{tabular}

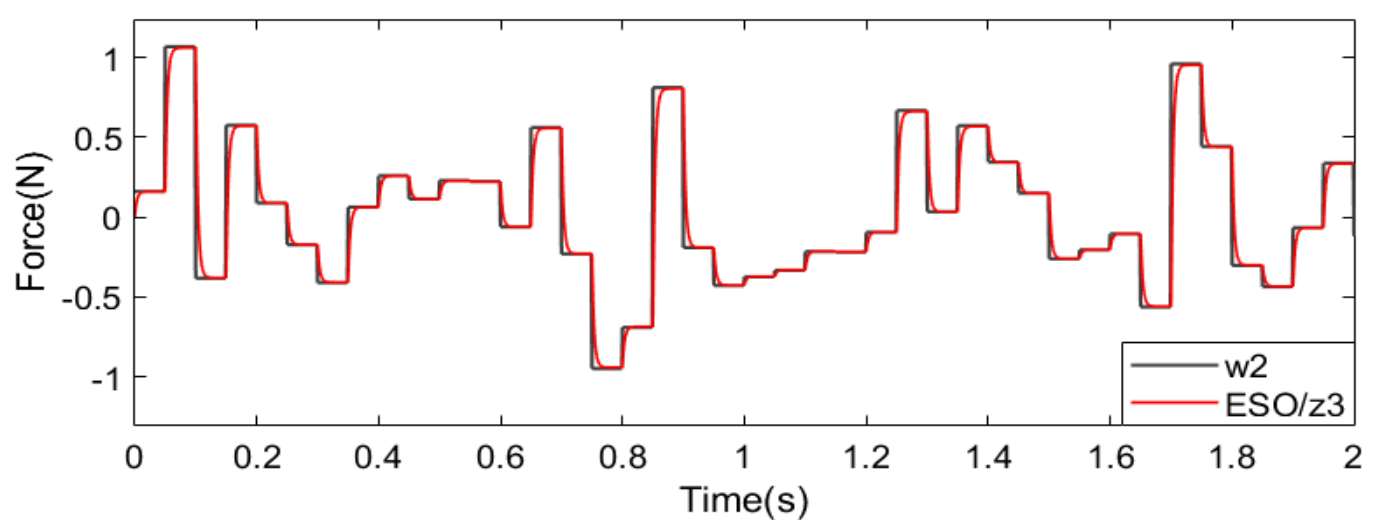

Fig. 5 Simulation of disturbances observation 


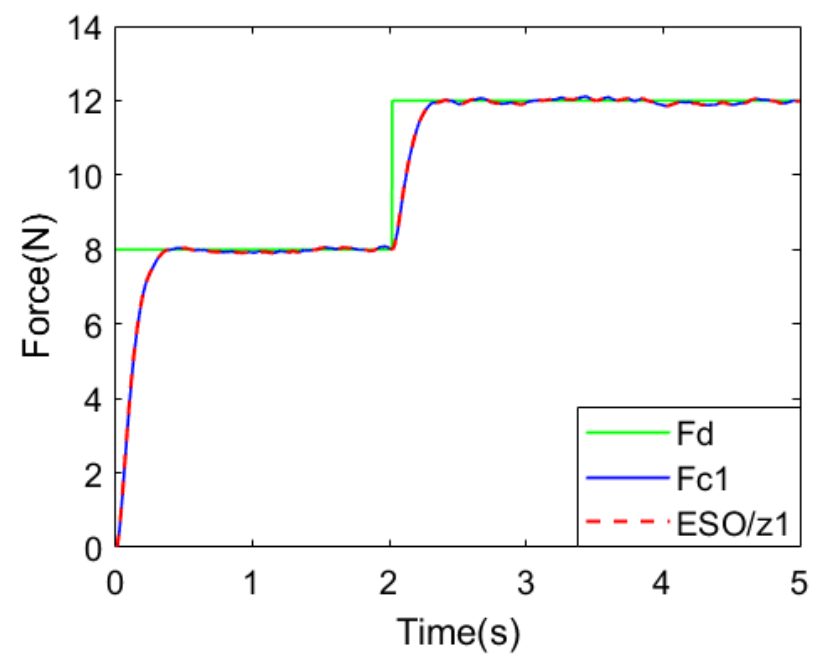

Fig. 6 Comparison of the actual output polishing force and the observation value In order to ensure the rapid response and control robustness of the controller. A feedback system ESO-based SMC and ADRC parallel control method is proposed. Add gaussian noise $w_{1}$ with a period of $0.001 \mathrm{~s}$ to the expected polishing force signal. Add a disturbance signal Band-Limited White Noise $w_{2}$ in the system state space. The Noise power is 0.01 and with a period of $0.01 \mathrm{~s}$. When the contact time is $0-2 \mathrm{~s}$, the expected polishing force is $8 \mathrm{~N}$. When contact time is $2-5 \mathrm{~s}$, the expected polishing force is $12 \mathrm{~N}$. Fig. 7 shows the simulation comparison of PID and SMC+ADRC control effects. Traditional PID responds quickly, with a response time of about $0.28 \mathrm{~s}$. But there is overshoot, and its robustness is lower than that of SMC+ADRC. Fig. 8 shows the simulation comparison of FuzzyPID and SMC+ADRC control effect. The response time of FuzzyPID is about $0.72 \mathrm{~s}$, and its robustness is better than PID, but worse than SMC+ADRC. Fig. 9 shows the simulation comparison between ADRC and $\mathrm{SMC}+\mathrm{ADRC}$. The response time of ADRC is about $0.43 \mathrm{~s}$, and the response time of $\mathrm{SMC}+\mathrm{ADRC}$ is $0.37 \mathrm{~s}$. Considering the rapid response and control robustness, the $\mathrm{SMC}+\mathrm{ADRC}$ proposed in this paper is the best. SMC+ADRC controller parameters are shown in Table. 3.

Table. 3 SMC + ADRC controller parameters

\begin{tabular}{cccc}
\hline Parameter & value & parameter & value \\
\hline$w_{c}$ & 250 & $c$ & 0.5 \\
$h_{1}$ & 0.002 & $r$ & 31250 \\
$B$ & 1 & $c_{1}$ & 2 \\
$\Delta$ & 0.5 & $\varepsilon$ & 2 \\
$k_{h}$ & 5 & $D$ & 1 \\
\hline
\end{tabular}




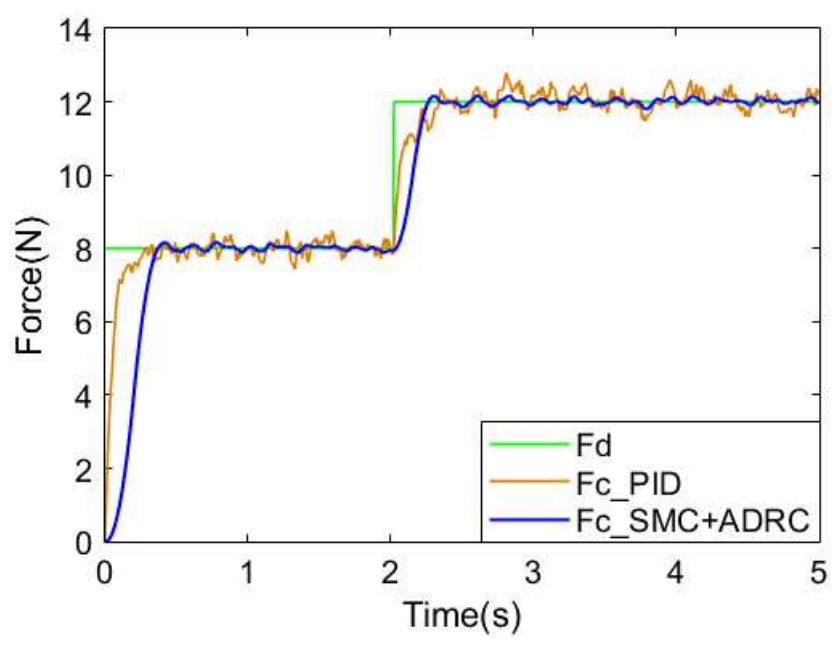

Fig. 7 Comparison of SMC+ADRC and PID control

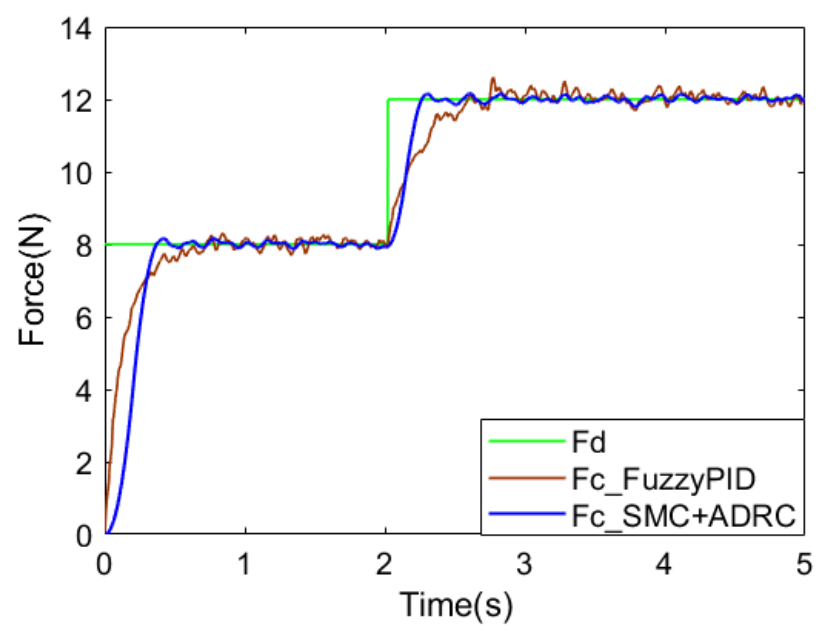

Fig. 8 Comparison of SMC+ADRC and FuzzyPID control

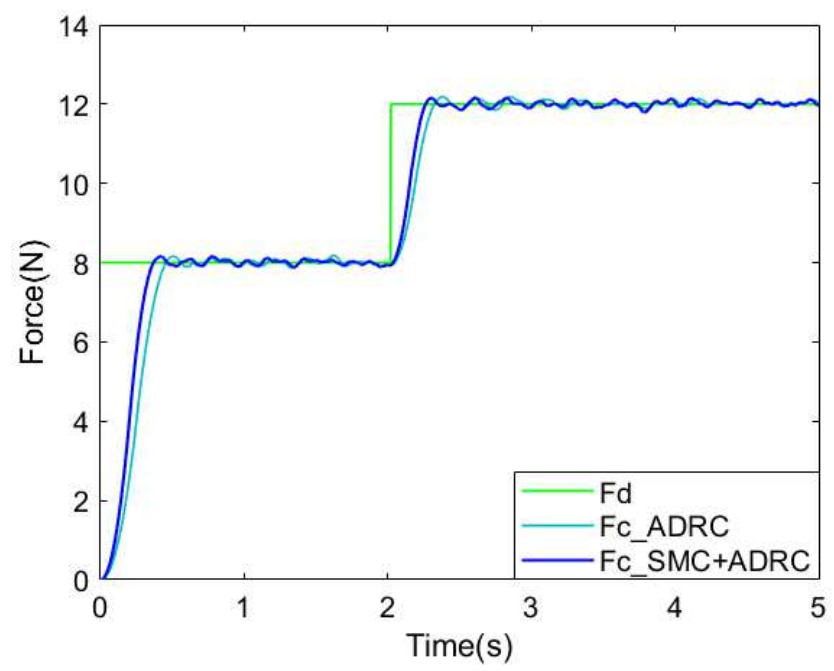

Fig. 9 Comparison of SMC+ADRC and ADRC control

The experiment uses Labview2015 to write control programs, including PID, FuzzyPID, ADRC, SMC+ADRC. The robot uses FANUCR-2000ic, the load is $210 \mathrm{Kg}$, and the repeated positioning accuracy is $\pm 0.05 \mathrm{~mm}$. Use Advantech IPC- $610-\mathrm{H}$ as the controller. Use Myrio module to complete real-time data collection. The brand and model of the 
six-dimensional force sensor is ATImin45. The brand and model of the tilt sensor is Ruifen HDA436T. The three-axis acceleration sensor is a Guanbiao 65-100 piezoelectric-type. Brand and model of electric proportional valve are Sintered Metal Company and ITV1050-312L. As shown in Fig. 10, the automatic polishing process device of the aero-engine blade. Fig. 11 shows the structure of aero-engine blade polishing end effector. The experiment using a rubber polishing wheel with a grit size is 1200 , and the air motor speed is $3500 \mathrm{r} / \mathrm{min}$.

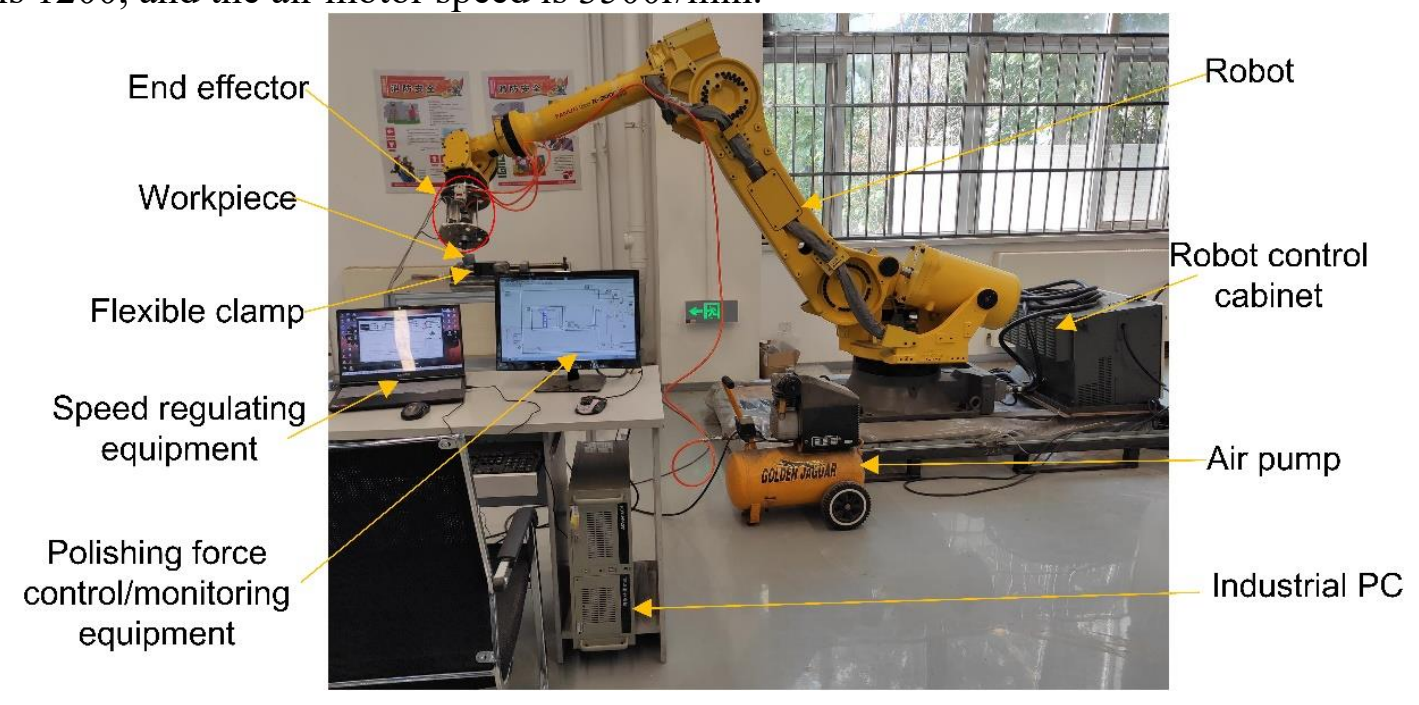

Fig. 10 Aero-engine blade automatic polishing experiment platform

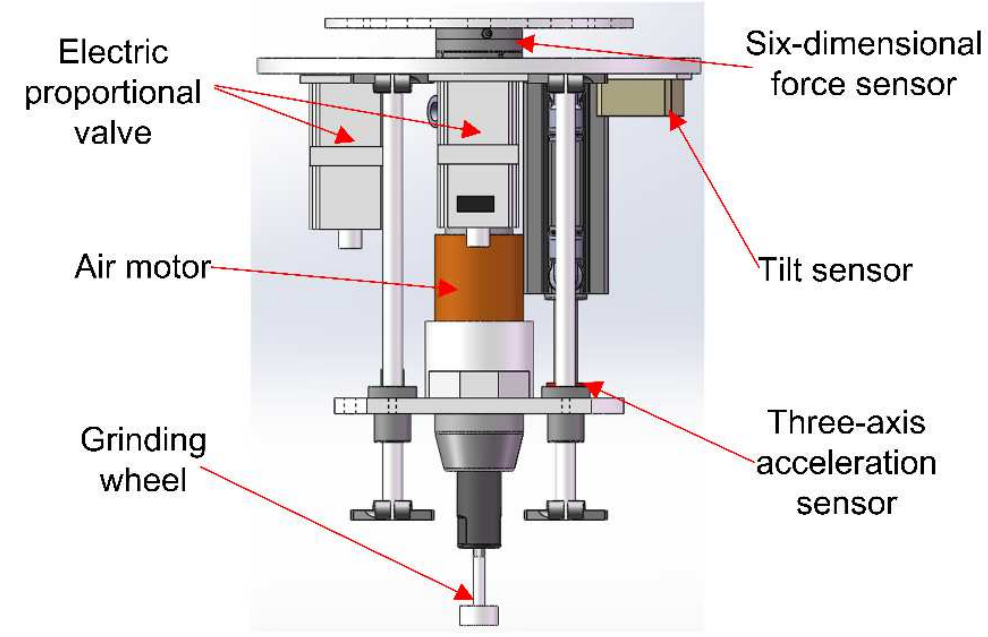

Fig. 11 End effector structure

The expected force is $8 \mathrm{~N}$ during the experiment. Collect the real-time control effect data of the polishing force to analyze the effectiveness of the SMC+ADRC method. Fig. 12 shows PID control result. The fluctuation range of polishing force is $6.25 \sim 9.75 \mathrm{~N}$. The amplitude of polishing force fluctuation is $3.5 \mathrm{~N}$. Fig. 13 shows FuzzyPID control result. Fluctuation range of the polishing force is $6.75 \sim 9.25 \mathrm{~N}$. The fluctuation amplitude of the polishing force is $2.5 \mathrm{~N}$. Fig. 14 shows the ADRC control result. Fluctuation range of the polishing force is $7.15 \sim 8.85 \mathrm{~N}$. Fluctuation amplitude of the polishing force is $1.7 \mathrm{~N}$. Fig. 15 shows the SMC+ADRC control result. Fluctuation range of the polishing force is $7.25 \sim 8.75 \mathrm{~N}$. Fluctuation range of the polishing force is $1.5 \mathrm{~N}$. The fluctuation amplitude of the polishing force is $0.75 \mathrm{~N}$. It 
can be seen from the above simulation results that the PID response is the fastest. But existing overshoot. Moreover, the robustness is lower than that of ADRC and fuzzy PID. Fuzzy PID has a slow response speed, but its robustness is relatively better. ADRC not only responds quickly but also has better robustness. However, due to the limited observation range of $\mathrm{ESO}$, the polishing force fluctuates greatly compared with the $\mathrm{SMC}+\mathrm{ADRC}$. It can be seen from the below experimental results. SMC+ADRC effectively suppresses the fluctuation of the polishing force during the polishing process. Fig. 16 shows the workpiece's micrography before finishing polishing. Fig. 17 shows the comparison of the actual machining effect under different control methods. Fig. 17(a) shows the Micrography of aero-engine blade using PID control. Fig. 17(b) shows the Micrography of aero-engine blade using FuzzyPID control. Fig17. (c) shows the Micrography of aero-engine blade using ADRC control. Fig. 17(d) shows the Micrography of aero-engine blade using SMC+ADRC control. Compare the surface quality after using different control methods by randomly selecting cross-sections. It can be seen from Fig. 16, that the maximum difference between the peak and the trough of the cross-section is 13.4um. It can be seen from Fig. 17(a). After PID control, that the maximum difference between the peak and the trough of the cross-section is 8.4um. It can be seen from Fig. 17(b). After FuzzyPID control, that the maximum difference between the peak and the trough of the aero-engine blade section is $5.8 \mathrm{um}$. It can be seen from Fig. 17(c). After ADRC control, that the maximum difference between the peak and the trough is 4.5um. Similarly, it can be seen from Fig. 17(d). After $\mathrm{SMC}+\mathrm{ADRC}$ control the maximum difference between the peak and the trough is 2.7um.

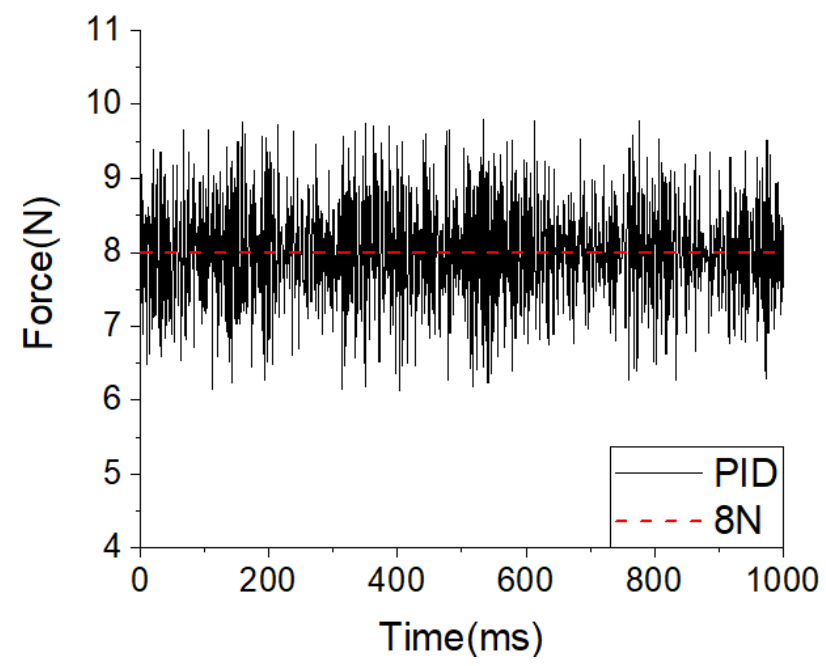

Fig. 12 Polishing force of PID control 


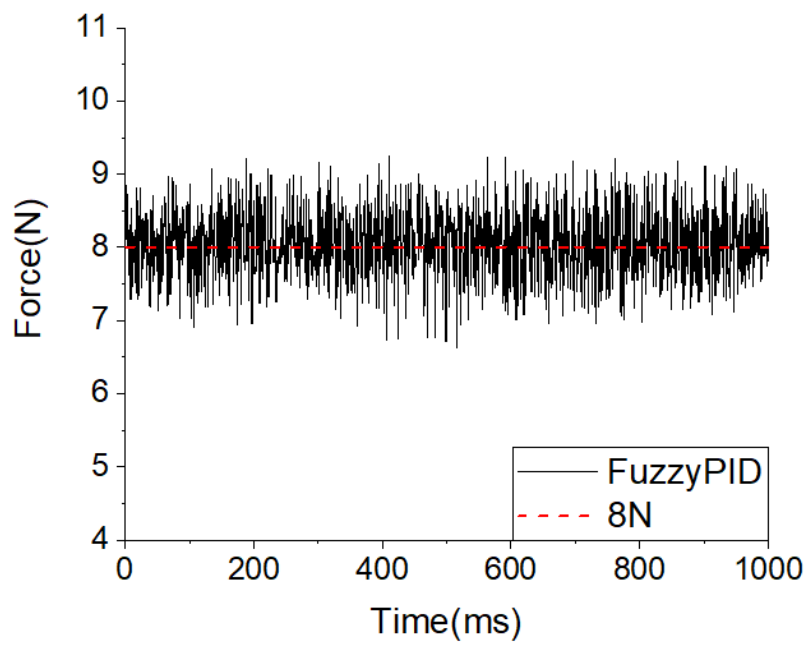

Fig. 13 Polishing force of FuzzyPID control

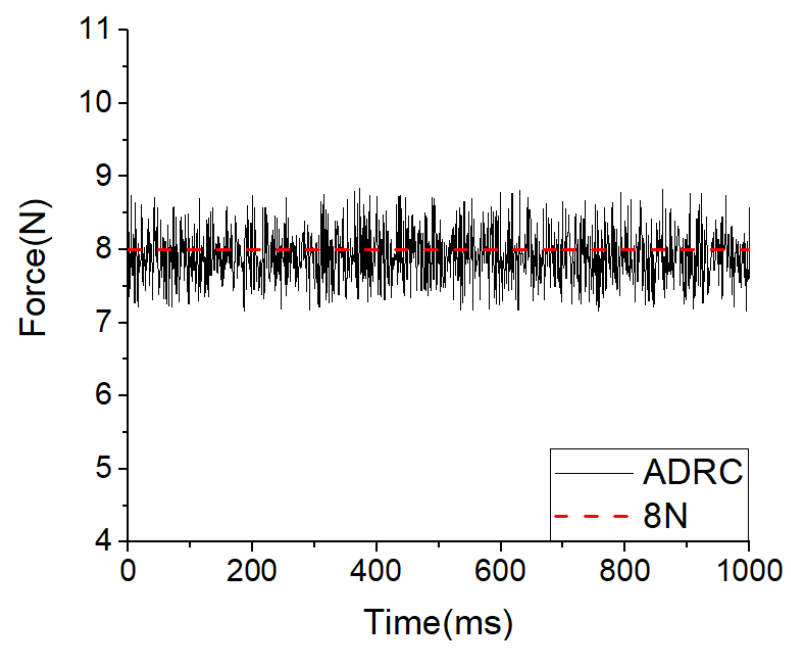

Fig. 14 Polishing force of ADRC control

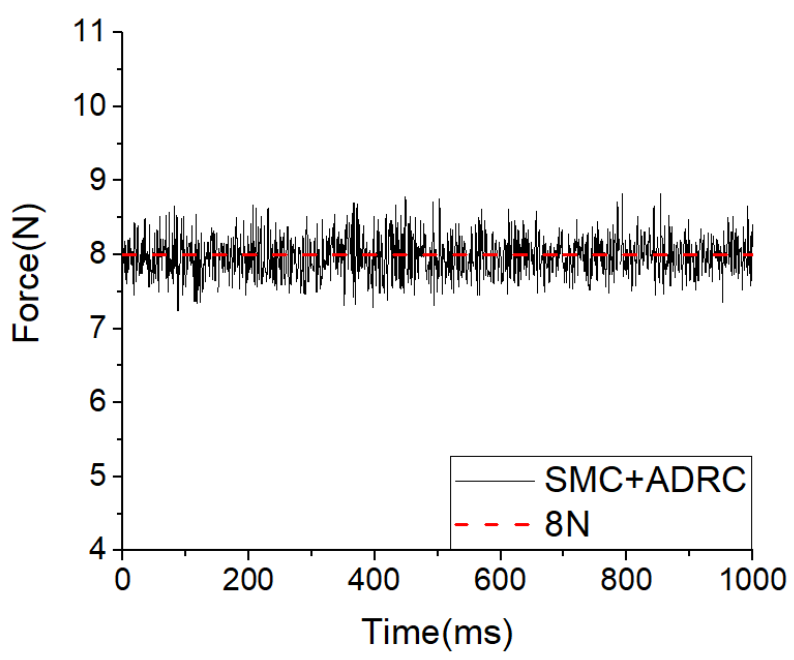

Fig. 15 Polishing force of SMC+ADRC control 

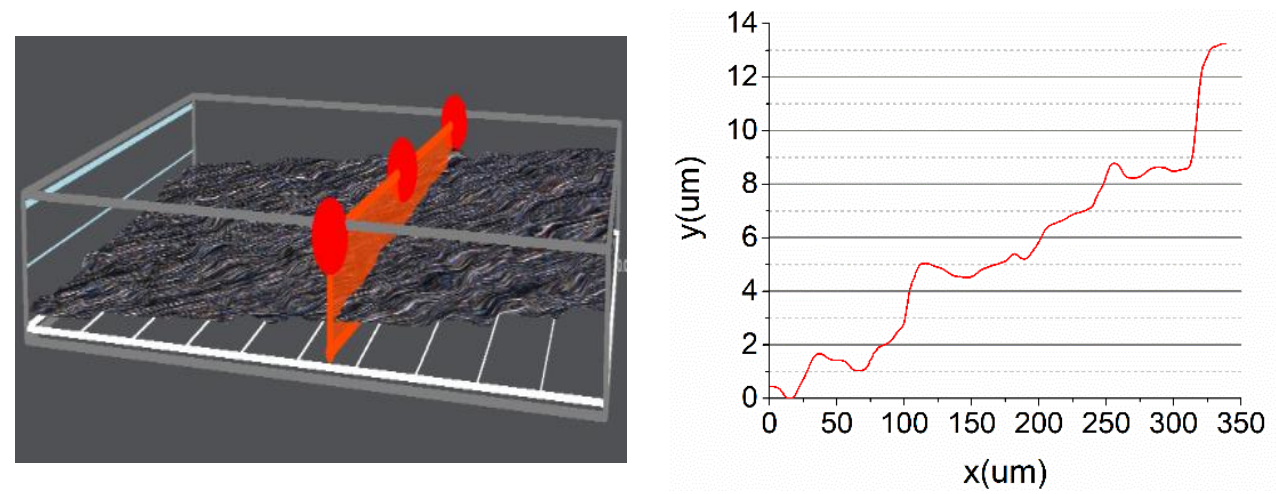

Fig. 16 Micrography of aero-engine blade before finishing polishing
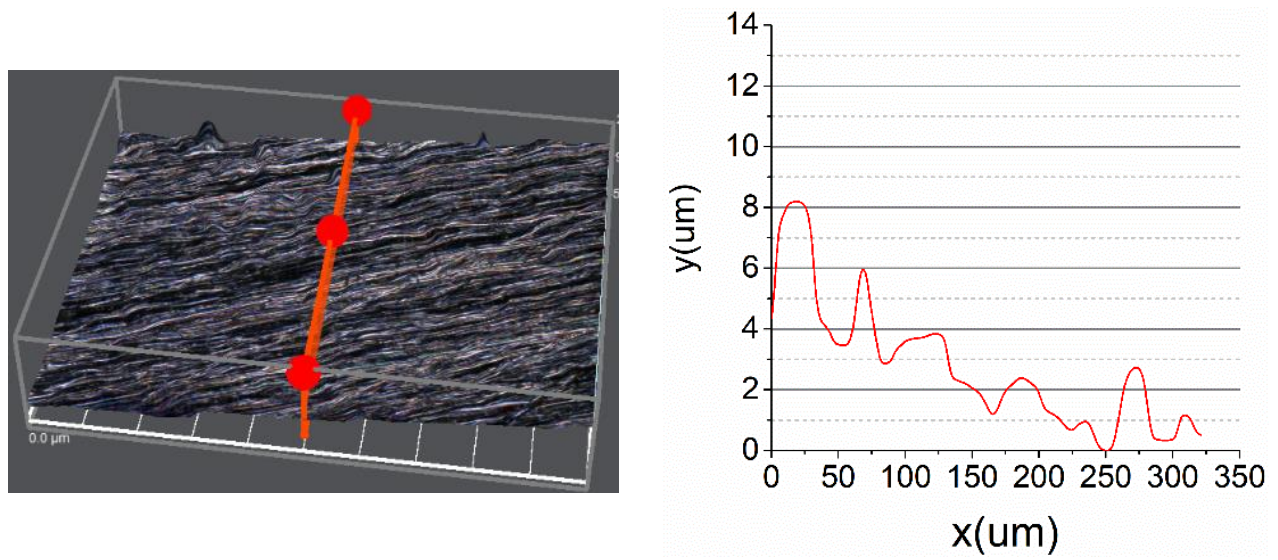

(a). Micrography of aero-engine blade effect under PID control
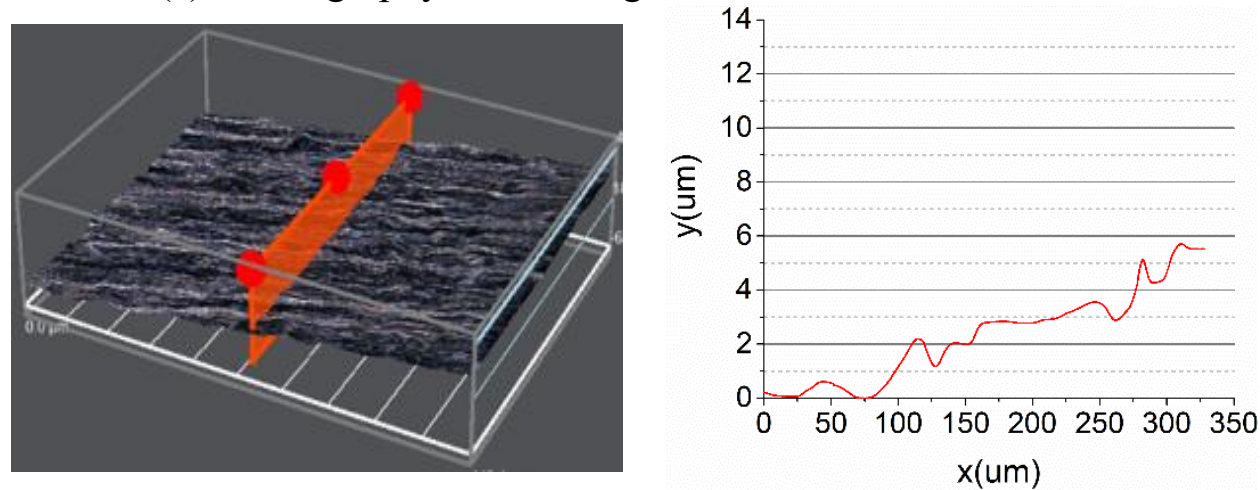

(b). Micrography of aero-engine blade effect under FuzzyPID control
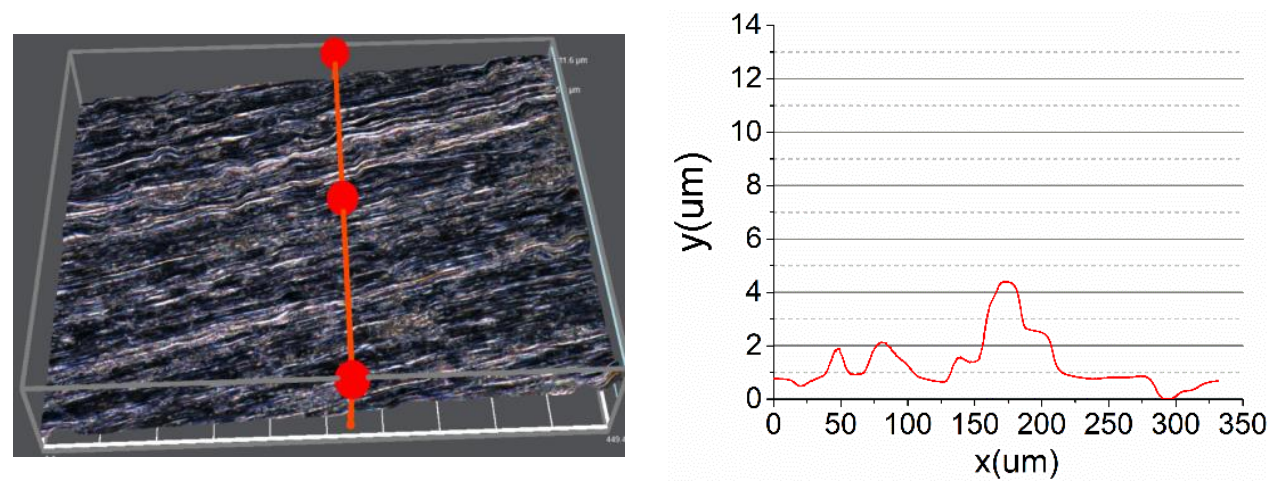

(c). Micrography of aero-engine blade effect under ADRC control 

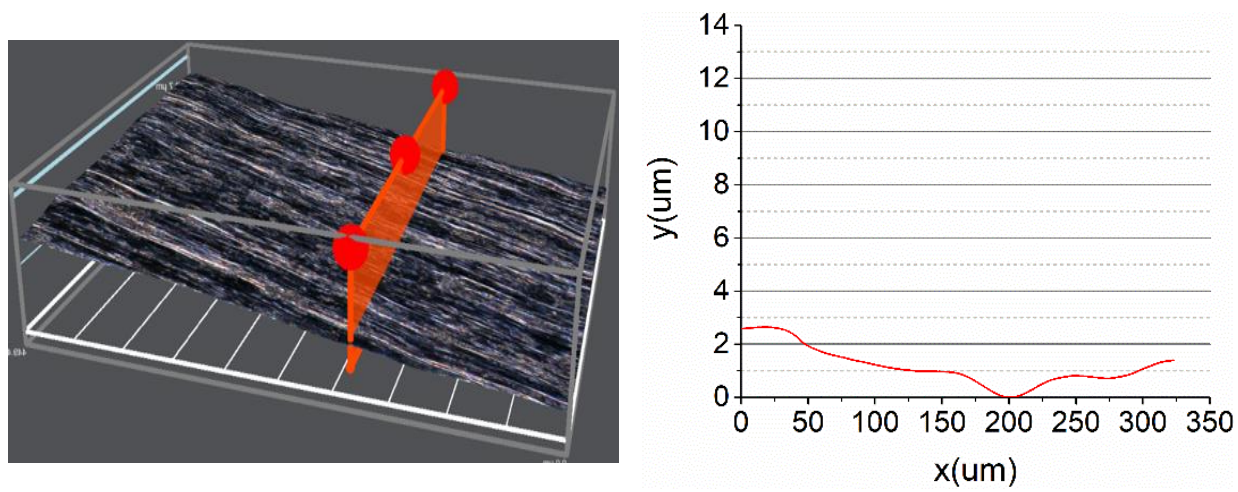

(d). Micrography of aero-engine blade effect under SMC+ADR control

Fig. 17 Comparison of polishing effects of different control algorithms

\section{Conclusion}

The polishing workload of aero-engine blades is heavy and the accuracy requirements are pretty high. However, due to the current imperfect robot force control algorithm result in the blades exist over-polishing or under-polishing. Therefore, it is significant to study maintenance the constant polishing force of aero-engine blades during the polishing process. At present, the constant force polishing control of aero-engine blade robots mainly focuses on feedback control based on system errors. However, the influence of environmental noise and modeling error on the polishing force of the system is time-varying. The current control based on system error feedback exist lag. Aiming to reduce the time-varying environmental noise and modeling error disturbance impact. This paper proposes an ESO-based SMC and ADRC parallel control method SMC+ADRC. Realize error feedback control after using this method to realize real-time compensation of disturbance. Meantime improving system control robustness and response rapidity. Ensures the constant polishing force in the process of polishing aero-engine blades.

Firstly, calibrate the six-dimensional force sensor, according to the maximum acceleration $a_{\max }$ of the end effector during the polishing process. Then build gravity compensation and zero-point drift compensation model. Use it to realize six-dimensional force sensor error compensation. After compensation, the average polishing force measure error of the is 0.1812 , and the variance is 0.2341 . The TD is used to realize the initial filtering of the polishing force, which can make the error of the polishing force within $-0.25 \sim 0.25 \mathrm{~N}$. ESO is used to realize the observation of environmental noise and system modeling error. Meantime realize real-time feedback compensation. According to the control boundary layer $\Delta$ divide the polishing force control system into two parallel control loops. When the switch value is within the control boundary error layer, use the ADRC control loop. When the switch is outside the control boundary error layer, use the ESO-base SMC control loop. Comparing with the traditional PID control, FuzzyPID control, and ADRC algorithms. Simulation and experimental proves that the proposed parallel control method SMC+ADRC with faster response and high robustness. Meantime, experimental results show application $\mathrm{SMC}+\mathrm{ADRC}$ can effectively suppress the fluctuation of polishing force, make the error of polishing force less than $0.75 \mathrm{~N}$, the surface roughness of blade is guaranteed to be less than $2.7 \mathrm{um}$. 


\section{Declarations}

Funding: This work was supported by National Key R\&D Program of China fund under Grant 2019YFB1311104.

Conflicts of interest/Competing interests: Not applicable.

Availability of data and material: All the data and material are availability

Code availability: All codes are availability

\section{Reference}

1. Huang H, Gong ZM, Chen XQ, Zhou L (2002) Robotic grinding and polishing for turbine-vane overhaul. Journal of Materials Processing Technology 2(127):140-145.

https://doi.org/10.1016/S0924-0136(02)00114-0

2. Huang H, Gong ZM., Chen XQ, Zhou L (2003) SMART Robotic system for 3D profile turbine vane airfoil repair. Int J Adv Manuf Technol 21(4): 275-283.

https://doi.org/10.1007/s001700300032

3. Fritz Klocke, Sein Leung Soo, Bernhard Karpuschewski, John A, Webster, et al (2015) Abrasive machining of advanced aerospace alloys and composites. CIRP Annals 64(2):581-604. https://doi.org/10.1016/j.cirp.2015.05.004

4. Shanawaz A. M, Sundaram S, Pillai U.T. S, Babu Aurtherson P (2011) Grinding of aluminium silicon carbide metal matrix composite materials by electrolytic in-process dressing polishing. Int J Adv Manuf Technol 57(1-4): 143-150. https://doi.org/10.1007/s00170-011-3288-4

5. Saleh Tanveer, Bishwas Indraneel, Rahman Mustafizur (2010) Efficient dressing of the wheel in ELID grinding by controllable voltage with force feed back. Int J Adv Manuf Technol 46(14):123-130. https://doi.org/10.1007/s00170-009-2076-x

6. He Qiuwei, Zhao Ji, Feng Mei, Zhang Chaopeng, Chen Hangde (2019) A study on the control strategies of a series-parallel hybrid platform for blade polishing. Int J Adv Manuf Technol 102(14):265-275. https://doi.org/10.1007/s00170-018-3064-9

7. Zhang Hongyao, Li Lun, Zhao Jibin, Zhao Jingchuan, Liu Sujie, Wu Jiajun (2020) Design and implementation of hybrid force/position control for robot automation grinding aviation blade based on fuzzy PID. Int J Adv Manuf Technol 107(3-4):1741-1754.

https://doi.org/10.1007/s00170-020-05061-y

8. Li Wenlong, Xie He, Zhang Gang, Yan Sijie, Yin Zhouping (2016) 3D shape matching of a blade surface in robotic grinding application. Journal of Robotics \& Machine Learning 21(5):22942306. https://doi.org/10.1109/TMECH.2016.2574813

9. Zhang Xudong, Chen Hao, Yang Ning (2017) A structure and control design of constant force polishing end actuator based on polishing robot. Proceedings of the IEEE nternational conference on information and automation, 18-20 July 2017, Macau, China.

https://doi.org/10.1109/ICIn-fA.2017.8079007

10. Chen Zhen, Shi Yaoyao, Lin Xiaojun, Zhang Junfeng, Chao Kang, et al (2019) A profileadaptive compliant polishing tool for aero-engine blade finishing process. Int $\mathbf{J}$ Adv Manuf Technol 102(9-12):3825-3838.

https://doi.org/10.1007/s00170-019-03477-9

11. Chen Zhen, Zhao Huan, Li Dingwei, Chen Lin, Tan Chao, et al (2019) Robotic grinding of a blisk with two degrees of freedom polishing force control. Int J Adv Manuf Technol 101(1- 
4):461-474.

https://doi.org/10.1007/s00170-018-2925-6

12. Wen Shuhuan, Zhu Jinghai, Li Xiaoli, Chen Shengyong (2014) A double-loop structure in the adaptive generalized predictive control algorithm for control of robot end-point contact force. ISA TRANSACTIONS 53(5):1603-1608.

https://doi.org/10.1016/j.isatra.2014.05.024

13. Xiao MuBang, Ding Ye, Fang Zaojun, Yang Guilin (2020) Contact force modeling and analysis for robotic tilted-disc polishing of freeform workpieces. Precision Engineering-Journal of The International Societies For Precision Engineering and Nanotechnology. 66:188-200.

https://doi.org/10.1016/j.precisioneng.2020.04.019

14. Wu Dongbo, Wang Hui, Zhang Kaiyao, Lin Xiaojun (2019) Research on flexible adaptive CNC polishing process and residual stress of blisk blade. Int J Adv Manuf Technol 103(5-8):2495-2513. https://doi.org/10.1007/s00170-019-03464-0

15. Loris Roveda, Nicola Pedrocchi, Federico Vicentini, Tosatti Lorenzo Molinari (2016) An interaction controller formulation to systematically avoid force overshoots through impedance shaping method with compliant robot base. Mechatronics 39:42-53.

https://doi.org/10.1016/j.mechatronics.2016.08.001

16. Loris Roveda, Nicola Pedrocchi, Lorenzo Molinari Tosatti (2016) Exploiting impedance shaping approaches to overcome force overshoots in delicate interaction tasks. International Journal of Advanced Robotic Systems 13(5). https://doi.org/10.1177/1729881416662771

17. Loris Roveda, Giacomo Pallucca, Nicola Pedrocchi, Francesco Braghin, Lorenzo Molinari Tosatti (2018) Iterative learning procedure with reinforcement for high-accuracy force tracking in robotized Tasks. IEEE Transactions on industrial informatics 14:1753-1763.

https://doi.org/10.1109/TII.2017.2748236

18. Xu Xiaohu, Zhu Dahu, Zhang Haiyang, Yan Sijie, Ding Han (2019) Application of novel force control strategies to enhance robotic abrasive belt grinding quality of aero-engine blades. Chinese Journal of Aeronautics 32:2368-2382. https://doi.org/10.1016/j.cja.2019.01.023

19. Du Huapeng, Sun Yuwen, Feng Deyang, Xu Jinting (2015) Automatic robotic polishing on titanium alloy parts with compliant force/position control. Proceedings of the Institution of Mechanical Engineers, Part B: Journal of Engineering Manufacture 229:1180-1192.

https://doi.org/10.1177/0954405414567518

20. Xiao Guijian, Huang Yun (2015) Constant-load adaptive belt polishing of the weak-rigidity blisk blade. Int J Adv Manuf Technol 78:1473-1484.

https://doi.org/10.1007/s00170-014-6724-4

21. Niu Dan, Chen Xisong, Yang Jun, Zhou Xingpeng (2019) Disturbance rejection control for raymond mill grinding system based on disturbance observer. Journal Of Central South University 24(9):2019-2027.

https://doi.org/10.1007/s11771-017-3611-9

22. Philippe Hamelin, Pascal Bigras, Julien Beaudry, Pierre-Luc Richard, Michel Blain (2011) Discrete-time state feedback with velocity estimation using a dual observer application to an underwater direct-drive grinding robot. IEEE-ASME Transactions On Mechatronics 17(1):187191. https://doi.org/10.1109/TMECH.2011.2154338

23. Philippe Hamelin, Pascal Bigras, Julien Beaudry, Pierre-Luc Richard, Michel Blain (2014) Multiobjective optimization of an observer-based controller: theory and experiments on an 
underwater grinding robot. IEEE Transactions On Control Systems Technology 22(5):1875-1882. https://doi.org/10.1109/TCST.2013.2296355

24. Chen X.S, Yang J, Li S.H, Li, Q (2009) Disturbance observer based multi-variable control of ball mill grinding circuits. Journal Of Process Control 19(7):1205-1213.

https://doi.org/10.1016/j.jprocont.2009.02.004

25. Yang Jun, Li Shihua, Chen Xisong, Li Qi (2010) Disturbance rejection of ball mill polishing circuits using DOB and MPC. Power Technology 198(2):219-228.

https://doi.org/10.1016/j.powtec.2009.11.010.

26. Dai Shijie, Li Shining, Ji Wenbin, Sun Zhenlin, Zhao Yufeng (2021) Force tracking control of grinding end effector based on backstepping plus PID. Industrial Robot-The International Journal of Robotics Research and Application. https://doi.org/10.1108/IR-10-2020-0229 